\title{
A Deep X-Ray Look at Abell 2142 - Viscosity Constraints From Kelvin-Helmholtz Eddies, a Displaced Cool Peak That Makes a Warm Core, and A Possible Plasma Depletion Layer
}

\author{
Qian H. S. Wang ${ }^{1}$ (1) and Maxim Markevitch ${ }^{2,3}$ (1) \\ ${ }^{1}$ Department of Astronomy, University of Maryland, College Park, MD 20742, USA \\ ${ }^{2}$ Astrophysics Science Division, NASA Goddard Space Flight Center, Greenbelt, MD 20771, USA \\ 3 Joint Space-Science Institute, University of Maryland, College Park, MD 20742, USA \\ Received 2018 July 6; revised 2018 October 4; accepted 2018 October 15; published 2018 November 19
}

\begin{abstract}
We analyzed $200 \mathrm{ks}$ of Chandra ACIS observations of the merging galaxy cluster A2142 to examine its prominent cold fronts in detail. We find that the southern cold front exhibits well-developed Kelvin-Helmholtz (KH) eddies seen in the sky plane. Comparing their wavelength and amplitude with those in hydrodynamic simulations of cold fronts in viscous gas, and estimating the gas tangential velocity from centripetal acceleration, we constrain the effective viscosity to be at most $1 / 5$ of Spitzer isotropic viscosity, but consistent with full Braginskii anisotropic viscosity for magnetized plasma. While the northwestern front does not show obvious eddies, its shape and the structure of its brightness profile suggest $\mathrm{KH}$ eddies seen in projection. The southern cold front continues in a spiral to the center of the cluster, ending with another cold front only $12 \mathrm{kpc}$ from the gas density peak. The cool peak itself is displaced $\sim 30 \mathrm{kpc}$ from the brightest cluster galaxy (BCG) (the biggest such offset among centrally peaked clusters), while the X-ray emission on a larger scale is still centered on the BCG, indicating that the BCG is at the center of the gravitational potential and the cool gas is sloshing in it. The specific entropy index of the gas in the peak $\left(K \approx 49 \mathrm{keV} \mathrm{cm}^{2}\right)$ makes A2142 a rare "warm core"; apparently the large displacement of the cool peak by sloshing is the reason. Finally, we find a subtle narrow, straight channel with a $10 \%$ drop in X-ray brightness, aligned with the southern cold front—-possibly a plasma depletion layer in projection.
\end{abstract}

Key words: galaxies: clusters: individual (A2142) - galaxies: clusters: intracluster medium $\mathrm{X}$-rays: galaxies: clusters

\section{Introduction}

The phenomenon known as a "cold front" was first discovered by Chandra in the galaxy clusters A2142 (Markevitch et al. 2000) (hereafter M00) and A3667 (Vikhlinin et al. 2001a, 2001b). Cold fronts are contact discontinuities in the density and temperature of the intracluster gas, seen in the sky plane as sharp edges (discontinuities of the gradient) of the $\mathrm{X}$-ray brightness, usually unresolved even with the Chandra angular resolution (for a review see Markevitch \& Vikhlinin 2007, hereafter MV07). Cold fronts may look similar to shocks in cluster X-ray images, but the gas temperature jump has the opposite sign-in the cold front, the temperature is lower on the denser side, so the two sides are near (though not exactly in) pressure equilibrium. Unlike in shock fronts, there is no flow of gas across the cold front, but there is often a tangential velocity difference.

Cold fronts can form during a merger as a result of ram pressure stripping of the infalling subcluster (the original proposal for A2142 in M00). Clear examples of such fronts are the Bullet subcluster (Markevitch et al. 2002) and the infalling galaxy NGC 1404 (Machacek et al. 2005; Su et al. 2017). Another class of cold fronts is observed in or near most cool cores, often as multiple concentric edges in a spiral pattern. These edges are caused by an off-axis subcluster merger and the resulting displacement of the dense core gas from the minimum of the gravitational potential, which sets off longlasting sloshing of that gas in the potential well (Markevitch et al. 2001; Ascasibar \& Markevitch 2006; hereafter A06; MV07). Such fronts are found in most cool cores (Ghizzardi et al. 2010), even in otherwise relaxed clusters; examples are
RXJ1720.1 + 26 (Mazzotta et al. 2001), A2029 (Clarke et al. 2004), Ophiuchus (A06; Million et al. 2010; ZuHone et al. 2010; Werner et al. 2016a; A496 (Dupke et al. 2007), Perseus (Churazov et al. 2003; Simionescu et al. 2012), Virgo (Simionescu et al. 2010; Roediger et al. 2011; Werner et al. 2016b), and as we now believe (Tittley \& Henriksen 2005; MV07), A2142.

Both types of cold fronts can be used for interesting tests of the microphysics of the intracluster plasma (MV07). In particular, the abruptness of the temperature and density changes across the front strongly limits thermal conductivity and diffusion (Ettori \& Fabian 2000; Vikhlinin et al. 2001a; MV07), suggesting that magnetic field drapes around the front surface and insulate the front. Because the gas tangential velocity is discontinuous across the front, cold fronts should develop Kelvin-Helmholtz (KH) instabilities. As indeed observed in, e.g., A3667, Bullet, and NGC 1404, they lead to eventual dissolution of the sharp interface. The growth of $\mathrm{KH}$ instability (KHI) depends on-and therefore can be used to constrain - the plasma viscosity and the structure and strength of the magnetic fields (Vikhlinin et al. 2001b; MV07; Roediger et al. 2013b, hereafter R13), though separating these two stabilizing effects may not be straightforward (ZuHone et al. 2015). Evidence for cold fronts developing KHIs has been seen indirectly in the form of the multi-edge structure of the radial brightness profile and "boxy" shape of the fronts, both consistent with being $\mathrm{KH}$ eddies seen in projection (e.g., Virgo, A496, Roediger et al. 2012, 2013a; NGC 1404, Su et al. 2017; A3667, Ichinohe et al. 2017). Their existence has been used to place an upper limit on the plasma isotropic 
viscosity (that is, disregarding the effect of the magnetic fields) to be $\sim 10 \%$ of the Spitzer value. As shown by magnetohydrodynamic (MHD) simulations (ZuHone et al. 2015), in the context of sloshing cold fronts, the suppression of KHIs in a plasma with a magnetic field draping around the cold front, with anisotropic Braginskii viscosity, should be qualitatively similar to the effect of a 1/10 Spitzer isotropic viscosity.

So far, KH eddies in the plane of the sky have been seen only in A3667 (Mazzotta et al. 2002; Vikhlinin 2011; Ichinohe et al. 2017). A possible eddy has also been reported at a sloshing cold front in Perseus (Walker et al. 2017), although the Perseus core is full of active galactic nucleus (AGN) bubbles and that feature could also be one of those. Those are the ones that can provide the most unambiguous constraints on the plasma microphysics. In this paper, we present another example of a cold front that shows apparent $\mathrm{KH}$ eddies, the southern front in A2142, based on a deeper Chandra observation of the cluster core. In addition, we analyze a recently found cold front at a very small radius, as well as two other interesting effects: a cool peak displaced from the central galaxy, as well as a subtle channel in the cluster X-ray brightness - a phenomenon similar to that we have recently discovered in another cluster, A520 (Wang et al. 2016).

While we concentrate on the core of A2142, where we now observe three concentric cold fronts (at $r \approx 12-340 \mathrm{kpc}$ ), this cluster exhibits another cold front far outside the core, $1 \mathrm{Mpc}$ from the center (Rossetti et al. 2013c), outside the Chandra coverage. A set of multiple concentric fronts at such different radii indicates "an extreme case of sloshing," quoting the above authors. Interestingly, A2142 has a specific entropy in the gas density peak that makes it a relatively rare "warm core"intermediate between cool-core and non-cool-core clusters (Cavagnolo et al. 2009; Giacintucci et al. 2017). We will try to clarify if this can be related to the observed strong sloshing. A2142 also has a giant radio halo whose structure spatially correlates with the cold fronts on all scales (Venturi et al. 2017).

In Section 2, we describe our treatment of Chandra data, as well as spectral and imaging analyses. In Section 2.1, we describe the procedure we used to generate a wavelet enhanced temperature map of the cluster's central regions. In Section 3, we describe each of the three cold fronts in turn, including the displacement of the cool core from the brightest cluster galaxy (BCG) in Section 3.3. We then discuss in Section 4 our results in the context of constraining viscosity, and in Section 5 a possible plasma depletion sheet. Finally we summarize our results in Section 6.

At the cluster redshift of $z=0.089,1^{\prime \prime}$ is $1.66 \mathrm{kpc}$ for $h=$ 0.7 and $\Omega_{M}=0.3$. Unless otherwise stated, errors in the text are given at $90 \%$ confidence.

\section{X-Ray Data Analysis}

We combined the archival Chandra Advanced CCD Imaging Spectrometer (ACIS) observations with ObsID 5005, 15186,16564 , and 16565 , omitting for convenience the short (16 ks) data set analyzed in M00. ObsID 5005 (45 ks) was taken in 2005 (PI L. VanSpeybroeck) and had the cluster center in ACIS-I3; it has been analyzed by Owers et al. (2009) and Johnson (2011). The latter three (153 ks total) were taken in 2014 (PI: M. Markevitch) and centered the cluster in ACIS-S3. An image from these observations have been looked at in Walker et al. (2016). We processed the data using CIAO (v4.9.1) and CALDB (v4.7.7), with a standard event filtering procedure to mask bad pixels, filter by event grades, remove cosmic ray afterglows and streak events, and detector background events identified using the VFAINT mode data. The data were then checked for background flares using the 2.5-7 keV light curve in $1 \mathrm{ks}$ time bins in a cluster-free region, separately for the FI and BI chips. As a more sensitive check for faint flares, we also used the ratio of $2.5-7 \mathrm{keV}$ to 9.5-12 keV counts. There were no period with strong flares. The final data we used have a total exposure of $197 \mathrm{ks}$, which is $97 \%$ of the raw exposure.

We accounted for the background following Markevitch et al. (2003) and Hickox \& Markevitch (2006), using the blanksky data sets from CALDB. For ObsID 5005, we used the Period E data set with an exposure of 1.55 Ms. For ObsIDs 15186,16564 , and 16565, we used the Period F data set with an exposure of $800 \mathrm{ks}$. For both imaging and spectral analysis, the background was scaled by the ratio of the $9.5-12 \mathrm{keV}$ counts (separately for front-illuminated and back-illuminated chips), which corrects for the secular background rate variability. The $90 \%$ uncertainty of the $0.8-9 \mathrm{keV}$ quiescent background modeled in such a way is $3 \%$ (Hickox \& Markevitch 2006), so we vary the background by this amount and include the effect in quadrature in our temperature measurement errors. The ACIS readout artifact was modeled using make_readout_.bg ${ }^{4}$ and treated as an additional background component, as in M00. We identified point sources for exclusion from our analysis by visual inspection using the $0.8-4 \mathrm{keV}$ and $2-7 \mathrm{keV}$ images at different binning and smoothing scales.

Spectral analysis was performed in XSPEC (version 12.9.1p). Instrument responses for spectral analysis were generated as described in Vikhlinin et al. (2005). We used the CHAV tools to generate the PHA, ARF, and RMF files for each pointing and then combined the data products. PHA files from different pointings were coadded for each of the observed data, blank-sky background, and simulated readout background, while ARFs and RMFs were weighed by the counts in the $0.5-2 \mathrm{keV}$ band (where most of the events are) in the spectral extraction region.

A single-temperature fit to the whole cluster in a $4^{\prime}$ circle (0.4 Mpc, covers most of the S3 chip) centered on $(\alpha, \delta)=$ $(15: 58: 20.4,+27: 13: 52.7)$ (FK5, J2000), using the $0.8-9 \mathrm{keV}$ band and the apec $*$ wabs model, gives $T=8.0 \pm 0.1 \mathrm{keV}$, metal abundance $0.28 \pm 0.01$ (relative to Anders \& Grevesse $1989)$, and absorption column $N_{H}=(7.7 \pm 0.3) \times 10^{20} \mathrm{~cm}^{-2}$. The errors are formal errors from fitting with and the effect of the $3 \%$ uncertainty in the blank-sky background added in quadrature. The best-fit temperature and abundance are the same as those reported in M00, while the best-fit $N_{H}$ is double the value $3.8 \times 10^{20} \mathrm{~cm}^{-2}$ from the Leiden/Argentine/Bonn (LAB) Survey (Kalberla et al. 2005), which probably reflects the uncertainty of the ACIS calibration at the lowest energiesthe reason for our excluding $E<0.8 \mathrm{keV}$ from the fits. In the analysis below, we fix the abundance and $N_{H}$ to the clusterwide best-fit values.

To make the exposure-corrected images, we created exposure maps using Alexey Vikhlinin's tools, ${ }^{5}$ assuming the spectrum of a single-temperature plasma with best-fit parameters from the $4^{\prime}$-radius circle described above. These are images of effective exposure time that include vignetting and

\footnotetext{
4 http://cxc.harvard.edu/contrib/maxim/make_readout_bg

5 http://hea-www.harvard.edu/ alexey/CHAV/
} 
variations in detector efficiency. Varying the assumed temperature within the range found in the cluster would make little difference to the broadband exposure map, as the counts are dominated by those around the peak of the ACIS effective area at 1-2 keV. (For the narrow-band exposure maps used in Section 2.1 it matters even less.) We divided the coadded (in sky coordinates) background-subtracted count images by the coadded exposure maps to get the final flux images.

\subsection{Temperature Map of the Small-scale Structure}

To determine the nature of the X-ray structure in the cluster core, we derived a temperature map of the core by subtracting the smoother, large-scale emission component, in order to enhance the contrasts of the small-scale features - that is, to get closer to their true temperatures. Because the precise 3D geometry of the gas in this asymmetric cluster is unknown, such a map necessarily provides only a qualitative picture of the core of A2142.

The map shown in Figure 2(a) was derived following the method described in M00 (without the deprojection step) and Wang et al. (2016). We extracted six narrow-band images in the $0.8-1-1.5-2-4-6-9 \mathrm{keV}$ bands. The flux and error images were smoothed by wavelets prior to deriving the temperature map, using the same wavelet decomposition coefficients for all bands. A single-temperature thermal model was fitted for each pixel to the six flux values from the narrow-band images, fixing the absorption column and metal abundance to the cluster best-fit values. This resulted in a wavelet-smoothed temperature map.

Wavelet decomposition separates the structures in the image at different scales, and helps us qualitatively deproject the large-scale components. Unlike a smoothing scheme such as Gaussian smoothing, which blurs everything with a symmetric kernel, wavelet decomposition preserves the shapes and brightness contrast of interesting small-scale features while at the same time having a basis in the statistical significance of the structures selected by the algorithm. Using a method described in Vikhlinin et al. (1994, 1998), we extracted wavelet components (with the atrous kernel and scales increasing in geometric progression) from images binned to 1 .'5 pixels, on scales of $2.5,5,10,20,39$, and $78 \mathrm{kpc}$ (or 1.5, 3, 6, 12, 24, and $47^{\prime \prime}$ ). Point sources were remove from the images at each scale and the components were coadded. Error images were treated with the same procedure.

\section{Cold Fronts}

The $0.8-4 \mathrm{keV}$ A2142 image, at full resolution without any smoothing or enhancements, is shown in Figure 1. We see the two prominent brightness edges that are the first cold fronts reported in M00 (marked "southern" and "NW"). The current, much deeper image reveals that the southern front spirals inward and ends with another cold front (marked "inner"). The inner front has been noted by Johnson (2011) in the earlier Chandra data set. A temperature map of this structure (Figure 2(a)) confirms that the gas behind those brightness edges is cool, thus the cold front interpretation is correct. A closer look at the image reveals that the southern front branches in two, one branch apparently continuing with a similar low curvature to the east (where we will find an intriguing "channel," Section 5) and another one curving toward the center and the inner front. Such a pattern is predicted by hydrodynamic simulations of gas sloshing for the recently formed fronts (see, e.g., A06 and their Figure 7, panels
1.8-2.1 Gyr, or Figure 2 in ZuHone et al. 2015). At this stage, the fronts do not yet form a complete spiral pattern and still exhibit the remainder of the Rayleigh-Taylor instability that gives rise to cold fronts with successively smaller radii (A06).

It is not clear whether the NW front and its more distant opposite (Rossetti et al. 2013c, outside this Chandra image) are part of the same sloshing pattern as the inner two or they are caused by another disturbance. A closer look at Figure 1 and the unsharp-masked image in Figure 5(b), as well as the gradient image in Walker et al. (2016), hints at subtle filamentary brightness enhancements that start at the NW front and go inward, as if they were extensions of the southern front. While Walker et al. interpreted them as projected KHI of the $\mathrm{NW}$ front, they may instead be the structures surviving from the stage when the cool gas currently in the core detached from the NW front and sank inward. However, this speculation is beyond the statistical accuracy of the present data set.

The gas density peak, which is right under the inner cold front and is the location of the coolest gas (Figure 2(a)), is offset by $\approx 30 \mathrm{kpc}$ from the BCG, which is likely to be the center of the gravitational potential. We will discuss this in Section 3.3.

The southern cold front shows structure that resembles eddies of the KHI, predicted by hydrodynamic simulations with sufficient resolution. The NW front exhibits an interesting structure consistent with such disturbances as well. We will discuss the constraints on viscosity that we can place using these observations in Section 4. We start below with the necessary preparatory analysis of the fronts.

\subsection{Southern Front}

We selected a sector enclosing the sharp segment of the southern cold front, as shown in Figure 2(b), and extracted a surface brightness profile from the exposure-corrected image (Figure 2(c)) to model the 3D gas density across the front. Our model describes the density profile inside the cold front with a power law and outside the cold front with a beta model, with a density jump at the cold front:

$$
n(r)= \begin{cases}n_{0}\left(r / r_{J}\right)^{\alpha}, & r \leqslant r_{J} \\ \frac{n_{0}}{x}\left[\frac{1+\left(r / r_{c}\right)^{2}}{1+\left(r_{J} / r_{c}\right)^{2}}\right]^{\beta}, & r>r_{J} .\end{cases}
$$

Here, $r_{J}$ is the radius of the density jump, $x$ is the density jump factor, $n_{0}$ is the density on the inside of the jump, and $r_{c}$ is the core radius of the beta model. The model is centered at the center of curvature of this section of the cold front (it is close to the X-ray peak), and we assume spherical symmetry of the model (i.e., the same curvature of the front along the line of sight (LOS) as in the sky plane). The best-fit parameters are given in Table 1. The model fits the profile very closely, showing a sharp jump at the cold front (Figure 2(c)).

We then extracted spectra from regions in the same sector on both sides of the southern front and fitted their projected temperatures in XSPEC: $T_{\text {cold,proj }}$ from a $10^{\prime \prime}$ wide annular segment inside, and $T_{\text {hot,proj }}$ from a $15^{\prime \prime}$ wide annular segment outside, allowing $1^{\prime \prime}$ of clearance from the front position on either side. Using the APEC normalization, we determined the absolute density by comparing it with the model's emission measure $\int n_{H} n_{e} d V$, assuming $n_{e}=1.17 n_{H}$. To evaluate the $3 \mathrm{D}$ gas temperature inside the cold front, $T_{\text {cold,deproj, we scaled the }}$ best-fit model in the outside region by the ratio of our model's emission measure for the outside component that is projected 


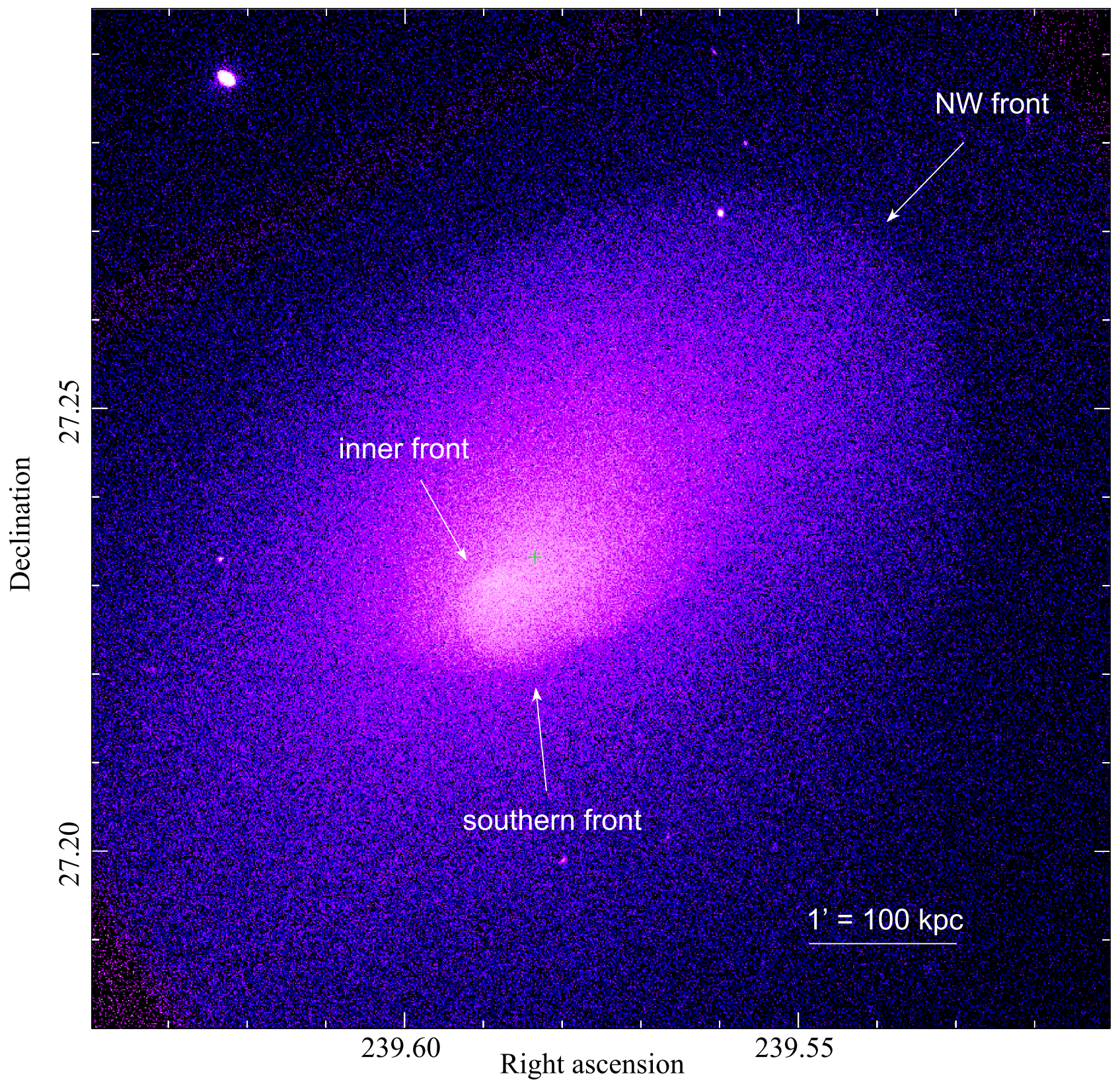

Figure 1. Broad view of the features we studied in A2142, shown by an unbinned 0.8-4 keV Chandra image (1 pixel is 0 "! 5). The cross marks the position of the BCG.

into the inner segment. We then refit the inner spectrum with this component added and held constant. Finally, we used XSPEC to check if $x$ and $n_{H, 0}$ needed to be corrected for the difference in $0.8-4 \mathrm{keV}$ emissivity in the presence of the temperature jump across the front (a small factor not included in the brightness profile fitting procedure). For the best-fit temperatures, the factor is $<0.1 \%$, so no correction was applied. The temperatures are given in Table 1. The gas pressure across the front is continuous within the $90 \%$ statistical uncertainties.

\subsection{Gas Velocity at the Southern Front}

For our instability analysis below, we now try to estimate the gas velocity at the front. Within the simple subcluster-stripping picture of the fronts, M00 used the pressure profile to constrain the velocity of the flow around the front, ascribing any difference in thermodynamic pressures across the front to ram pressure. They obtained a rough upper limit of $v<400 \mathrm{~km} \mathrm{~s}^{-1}$ for the southern front. A more accurate way to estimate the front velocity from the pressure profile is proposed in Vikhlinin et al. (2001a) for A3667. However, we now think that (at least) the southern and the inner fronts are, in fact, sloshing fronts with gas flowing tangentially (see, e.g., A06 for the possible flow patterns). In particular, the cool gas under the southern front is likely to be flowing from NW along the inward spiral.

In this picture, we can try to estimate the velocity of the curved tangential flow from the centripetal acceleration, as was done in Markevitch et al. (2001) and Keshet et al. (2010). In the simplest approximation, the outer gas is stationary while the cold front gas inside the front is in circular orbit with velocity $v$ in the cluster gravitational potential. Then,

$$
\frac{G M\left(r^{\prime}\right)}{r^{\prime 2}}=-\frac{1}{\rho} \frac{d p}{d r}+\frac{v^{2}}{r^{\prime}}
$$




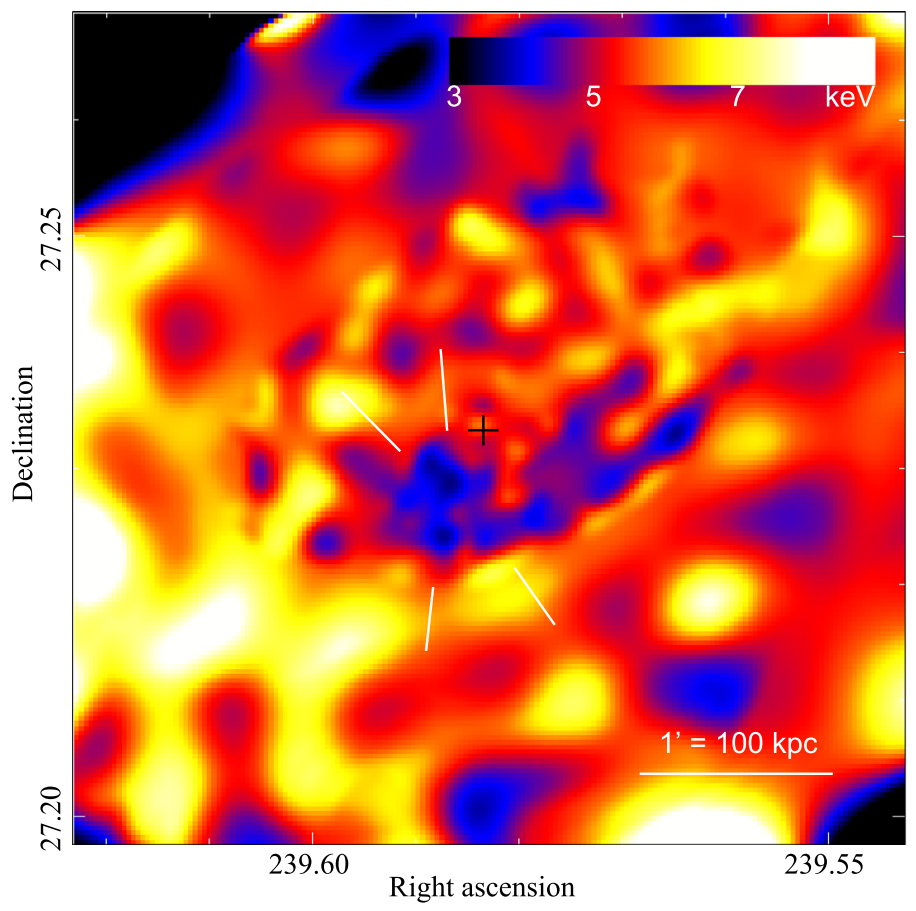

(a)

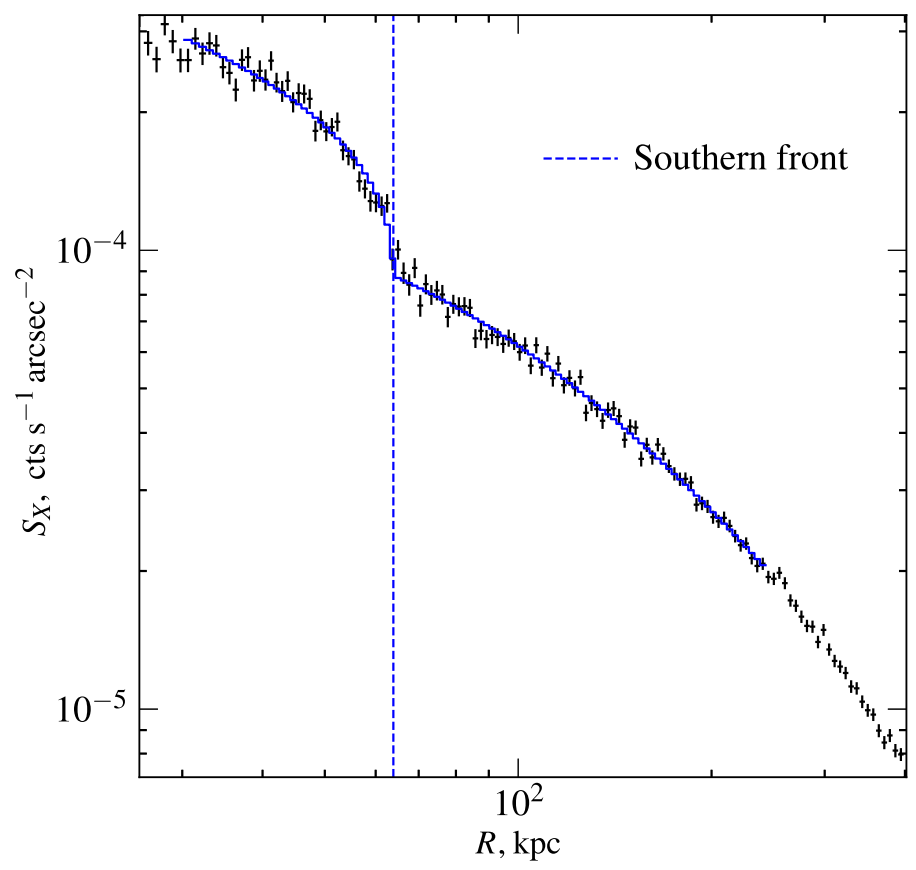

(c)

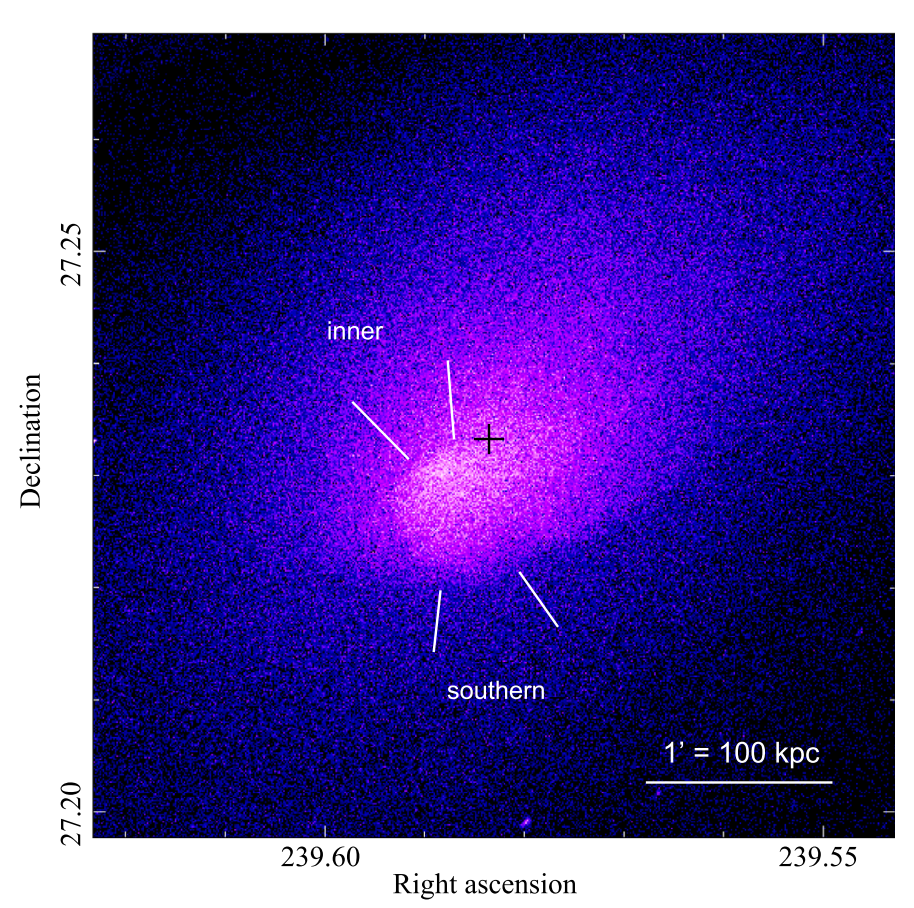

(b)

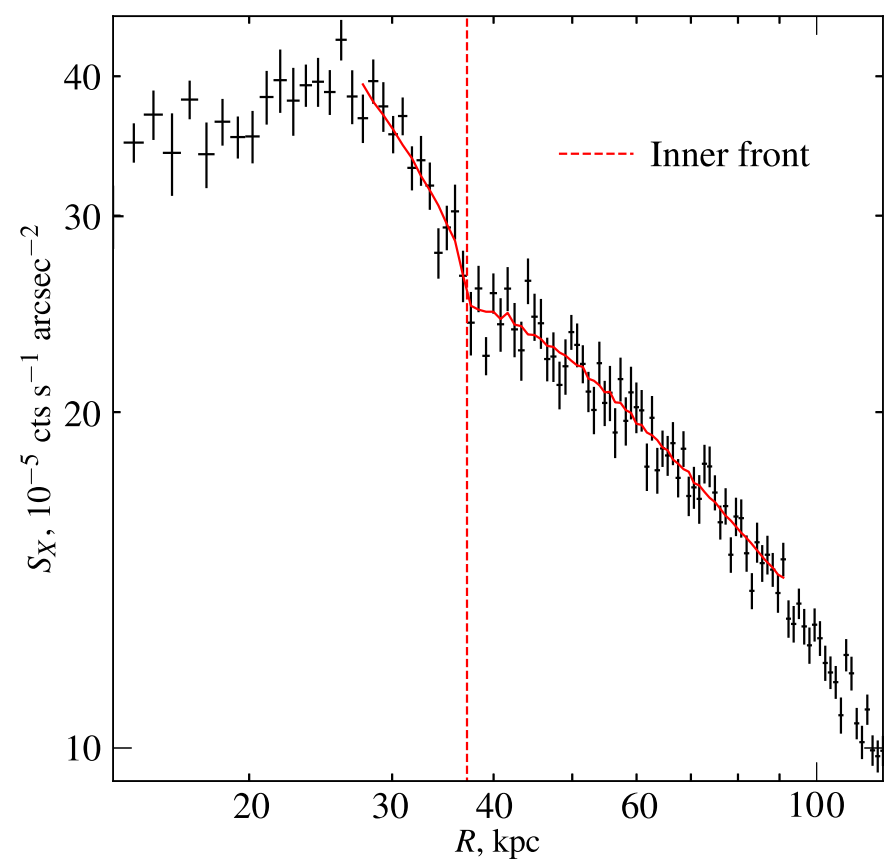

(d)

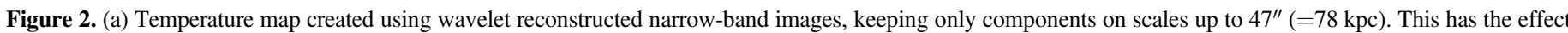

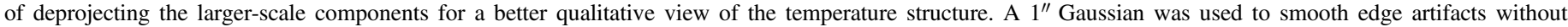

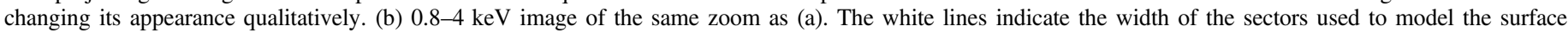

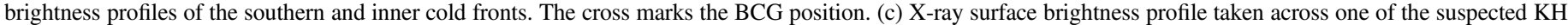

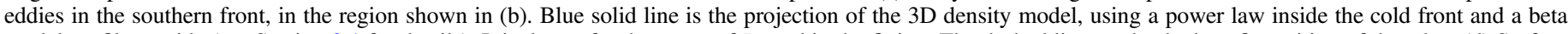

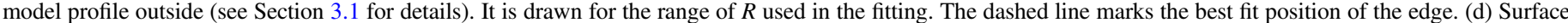

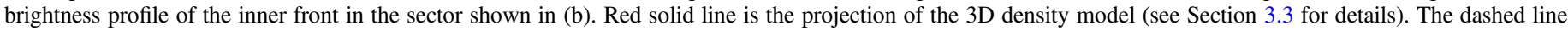
marks the best-fit position of the edge.

where $M$ is the cluster total mass within the radius $r^{\prime}, \rho$ is gas density, and $p$ is thermodynamic pressure. Here, the $r$ coordinate is from the center of the model density profile (the center of curvature of the cold front) and $r^{\prime}$ is from the center of mass (the BCG). At the cold front, they are at an angle of only $15^{\circ}$, so $d r^{\prime} / d r=0.97$ there, and we can ignore this distinction for an approximate estimate. The left-hand side of Equation (2) is continuous over the cold front, because the cluster total mass 
Table 1

Best-fit Cold Front Model Parameters

\begin{tabular}{|c|c|c|c|c|c|c|c|c|c|}
\hline Location & $\begin{array}{c}n_{H, 0} \\
10^{-3} \mathrm{~cm}^{-3}\end{array}$ & $\begin{array}{r}r_{J} \\
\mathrm{kpc}\end{array}$ & $x$ & $\alpha$ & $\beta$ & $\begin{array}{c}r_{c} \\
\mathrm{kpc}\end{array}$ & $\begin{array}{c}T_{\text {cold, proj }} \\
\mathrm{keV}\end{array}$ & $\begin{array}{l}T_{\text {cold, deproj }} \\
\quad \mathrm{keV}\end{array}$ & $\begin{array}{l}T_{\text {hot }} \\
\mathrm{keV}\end{array}$ \\
\hline Southern & $16.4 \pm 0.16$ & $63.9_{-0.5}^{+0.6}$ & $1.87 \pm 0.1$ & $-0.51 \pm 0.09$ & $-0.60_{-0.05}^{+0.04}$ & $75_{-15}^{+16}$ & $6.9_{-0.5}^{+0.8}$ & $5.8_{-0.9}^{+1.1}$ & $9.0_{-0.9}^{+1.1}$ \\
\hline NW1 & $4.31 \pm 0.04$ & $175.0 \pm 1.0$ & $2.14_{-0.10}^{+0.09}$ & $-0.42 \pm 0.04$ & $-0.71_{-0.06}^{+0.05}$ & $218_{-37}^{+39}$ & $8.6_{-0.8}^{+1.2}$ & $7.9_{-1.3}^{+1.6}$ & $10.5_{-1.2}^{+1.9}$ \\
\hline NW2 & $4.31 \pm 0.04$ & $174.6_{-1.7}^{+1.0}$ & $2.07_{-0.10}^{+0.11}$ & $-0.50 \pm 0.04$ & $-0.66 \pm 0.05$ & $178_{-43}^{+41}$ & $7.2_{-0.7}^{+0.9}$ & $6.1_{-0.9}^{+1.1}$ & $10.5_{-1.3}^{+2.2}$ \\
\hline
\end{tabular}

Note. $n_{H, 0}$ is given as the model density on the inside of the jump, calculated using the density and temperature of the outer component. Errors are $90 \%$.

distribution (dominated by dark matter) is smooth. However, the moving gas inside the cold front effectively feels a lower mass. Therefore, we can check for a difference in the total mass derived under the hydrostatic equilibrium assumption (e.g., Sarazin 1988) on the inside and outside of the cold front, and attribute it to the centripetal term. Using the gas density model of Equation (1) and the temperatures on the two sides derived above (assumed constant at those values on both sides), we calculated the difference between the second term in Equation (2) to be $(4.0 \pm 2.7) \times 10^{3} \mathrm{~km}^{2} \mathrm{~s}^{-2} \mathrm{kpc}^{-1}$, which corresponds to $\mathrm{a} \approx 35 \%$ drop in the apparent total mass on the inside of the cold front. The hydrostatic mass given by the outer part of the model (i.e., the true mass under our assumptions $)$ is $(1.5 \pm 0.3) \times 10^{13} M_{\odot}$ within $r^{\prime}=75 \mathrm{kpc}$ of the BCG.

This gives a tangential velocity of the cold gas of $(550 \pm 190) \mathrm{km} \mathrm{s}^{-1}$, where the errors are statistical and include the uncertainties of the parameters $\alpha, \beta, r_{J}, r_{c}, T_{\text {cold,deproj, and }}$ $T_{\text {hot }}$ (Table 1). If we use a smaller radius of curvature such as that of the cold front at this position, we get a lower value but not by much, because of the square root. Given the unknown 3D geometry and a number of assumptions, this is, of course, only a qualitative estimate with a factor of 2 accuracy at best. Furthermore, if the outer gas also rotates but in the opposite direction, one can in principle have a much higher relative tangential velocity and still satisfy Equation (2). While such a scenario is unlikely considering how the sloshing fronts formwe do not expect flows faster than Mach $\sim 0.3-0.5-\mathrm{a}$ conservative upper limit on the relative velocity is probably the sound speed in the outer gas $\left(1500 \mathrm{~km} \mathrm{~s}^{-1}\right)$, from the fact that we do not see any shocks immediately outside this cold front. We will use the velocity estimate of $550 \mathrm{~km} \mathrm{~s}^{-1}$ in Section 4 below.

\subsection{The Displaced Gas Peak and the Inner Front}

We noted above that the gas density peak is offset $\sim 30 \mathrm{kpc}$ from the position of the brightest cluster galaxy (Figure 1, Figure 2(b)). Such offsets are rare but not unknown-they have been seen, e.g., in A644 (Buote et al. 2005), Ophiuchus (Million et al. 2010; ZuHone et al. 2010; Hamer et al. 2012; Werner et al. 2016a), A1991 (Hamer et al. 2012), and Zw1742 +33 (Ettori et al. 2013). However, to our knowledge, this is the largest offset seen in a cluster that still has a cool density peak. A comparable offset of $20 \mathrm{kpc}$ is seen in $\mathrm{Zw} 1742+33$, but that cluster also shows evidence of AGN X-ray cavities emanating from its BCG, which has an active nucleus seen in the radio and X-rays. In comparison, the BCG in A2142 is currently very faint in the radio (Venturi et al. 2017) and is not detected in the X-ray; we see no evidence for X-ray cavities either. Thus, the offset peak that we observe is clearly the result of sloshing and of the merger that set it off.

We subtracted the cool sloshing structure from the X-ray image by wavelet decomposition to see the larger-scale X-ray gas distribution. After the subtraction of components $20 \mathrm{kpc}$ and smaller (using the same decomposition as Section 2.1), we are left with the image shown in Figure 3(a). The contours of the subtracted small-scale structure are overlaid. We see a symmetric elliptical X-ray structure centered very near the $\mathrm{BCG}$. This is consistent with a picture where the BCG is the center of the gravitational potential of the cluster, and the gas beyond the inner sloshing structure is largely in hydrostatic equilibrium with it (this does not exclude slower motions that can accompany the outer cold fronts). The gravitational lensing map of Okabe \& Umetsu (2008) does show the main mass peak of the cluster near this BCG. The second brightest galaxy seen in Figure 3(b), which was thought in M00 to be the center of a merging subcluster, appears not to be physically related to the cluster, based on its high peculiar velocity $\left(1840 \mathrm{~km} \mathrm{~s}^{-1}\right.$ from the BCG, Oegerle et al. 1995) and lack of a mass concentration (Okabe \& Umetsu 2008).

We will now model the inner cold front in order to derive the parameters of the gas in the offset density peak. A surface brightness edge near the peak of the X-ray emission spans a sector from east to north (Figure 2(b)). The contrast in X-ray brightness and projected temperature is highest in the northeastern quadrant, and the edge disappears to the west. It is a cold front, as shown by the temperature map (Figure 2(a)). We extracted a brightness profile (Figure 2(d)) in the sector shown in Figure 2(b) and model it it as follows. The density profile inside the edge is centered on the center of curvature of the front and is a power law. The outer gas is modeled with an ellipsoidal component following a power-law profile, centered on the BCG. The ellipticity of the outer component model is achieved simply by rescaling the coordinate of the long axis before calculating the model density in 3D. Both the position angle and ellipticity of the outer component were deduced from the X-ray brightness contours of the remaining cluster emission after we subtracted the core structure (as described above), and fixed during the fit. Since the two density components have different centers, we could not just calculate a 1D projected model. Instead, we projected the model onto the same image plane as the flux image and extracted a brightness profile in the same sector. The best-fit model is shown in Figure 2(d). To determine a deprojected central temperature, we first fitted the spectrum extracted from a sector, $17 \mathrm{kpc}$ wide, just outside the cold front. Then, we created an image of the ellipsoidal component with a spherical cutout for the core and used it to normalize the projected contribution to an inner sector, $10 \mathrm{kpc}$ 

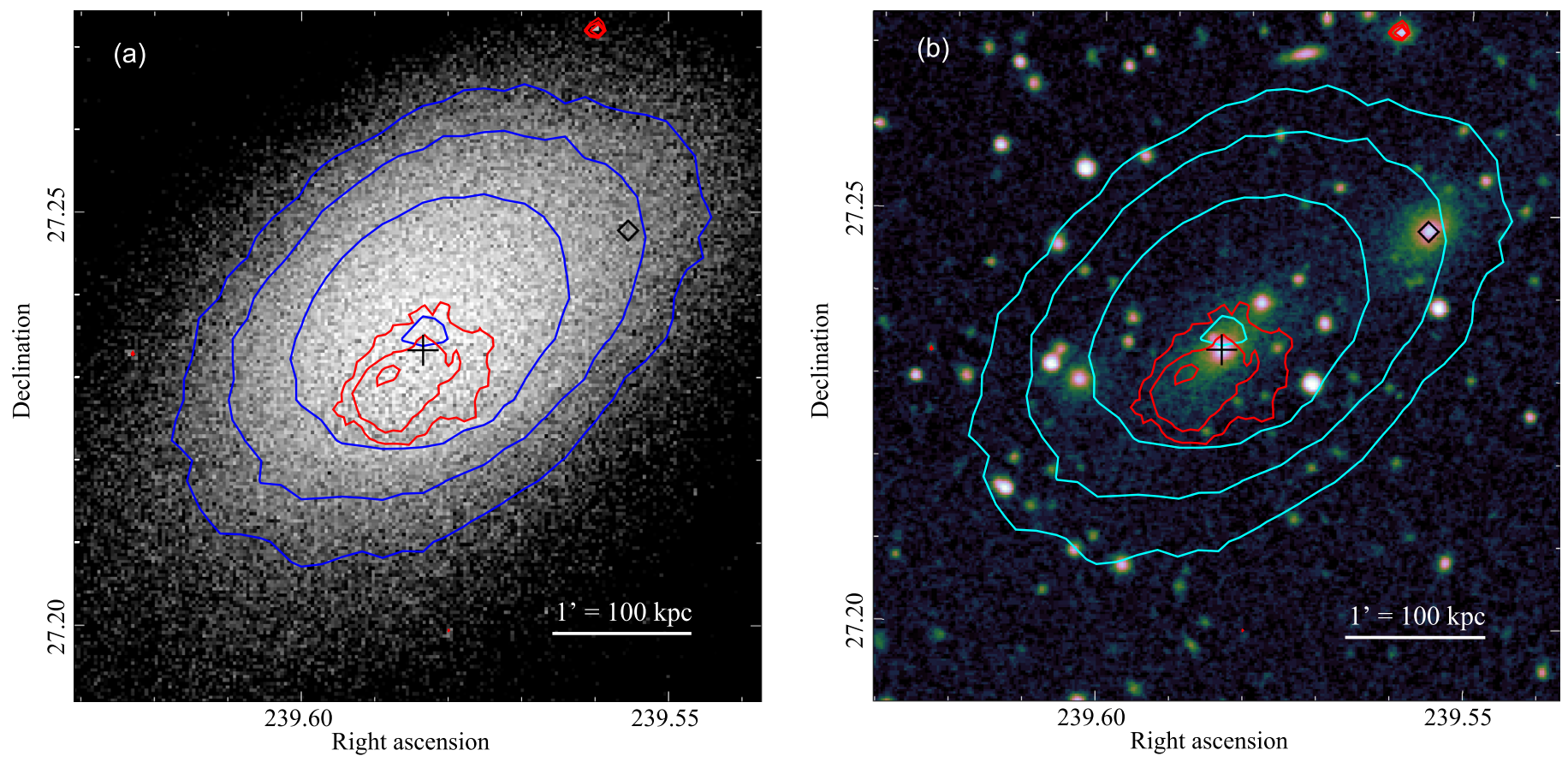

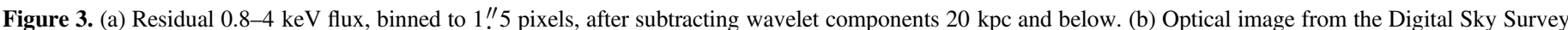

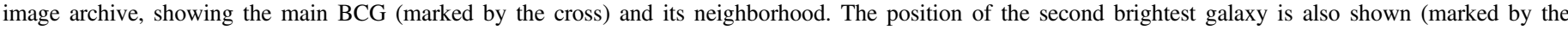

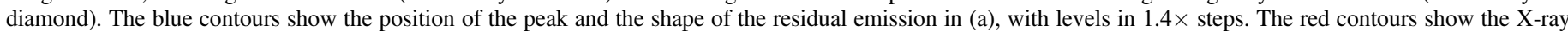

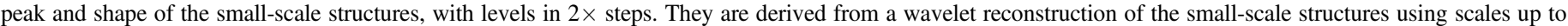
$39 \mathrm{kpc}$. The wavelet reconstruction uses the same decomposition as that described in Section 2.1.

thick, inside the front. We then fixed this contribution at the best-fit outer temperature and fit the inner temperature. Finally, we use the APEC model normalization to derive the gas densities in 3D as we did in Section 3.1.

Our deprojected density just behind the cold front (near the peak) is $n_{H} \approx 2.3 \times 10^{-2} \mathrm{~cm}^{-3}$ and temperature $T=4.0_{-0.6}^{+0.8} \mathrm{keV}$. The gas specific entropy index, commonly defined in the cluster field as $K=T_{e} n_{e}^{-2 / 3}$, is $K \approx 49 \mathrm{keV} \mathrm{cm}^{2}$ (statistical errors are probably meaningless because the systematic uncertainties dominate). The true value at the peak can be slightly lower because our spectral fitting region does not resolve the peak. For the gas immediately outside this cold front, our model gives $K \approx$ $120 \mathrm{keV} \mathrm{cm}^{2}$. We note that our value for the central entropy index is lower than $58 \pm 2 \mathrm{keV} \mathrm{cm}{ }^{2}$ in Giacintucci et al. (2017) from the same data set; however, the difference is expected because those authors used a different definition of "central entropy" in order to be consistent with Cavagnolo et al. (2009), who combined the projected temperature with the 3D gas density, whereas both our quantities are deprojected.

The above small difference notwithstanding, our value of the central entropy places A2142 in the gap between the cool-core and non-cool-core clusters (Cavagnolo et al. 2009). This is apparently related to strong sloshing in this cluster. As shown by ZuHone et al. (2010), sloshing of a cool core can balance radiative cooling, except for the very central region, by facilitating mixing with the higher-entropy gas from outside the core. Once the gas peak is displaced from the minimum of the gravitational potential, it becomes even more prone to mixing, because it expands (which reduces the density contrast) and because the stabilizing effect of gravity is removed. We may have caught A2142 at the moment of dissolution of its former cool core by sloshing. The displacement of the gas peak should also have deprived the $\mathrm{cD}$ galaxy of the accreting cool gas for a significant period of time, which is why it does not exhibit an AGN, similarly to Ophiuchus and to most clusters without cool cores.

\subsection{NW Front}

Upon close inspection, the NW front (Figure 4) shows interesting structure, which includes a "boxy" shape and apparent multiple edges at its nose. We extract brightness profiles in sectors NW1 and NW2 as shown in Figure 4(a) and fit them as described in Section 3.1 with the density model given in Equation (1), centered on the front center of curvature (same for both sectors). The best-fit parameters, along with the gas temperatures across the front, are given in Table 1. For the observed temperature jumps, a $1 \%$ reduction was applied to the jump factor to correct for the higher $0.8-4 \mathrm{keV}$ emissivity at the lower deprojected temperature. These two segments of the cold front are visually similar, have the same radius of curvature, and the brightness jump can be traced by the same circle. Their model parameters are therefore very comparable, and indeed their best-fit density jump positions, jump factors, and outer model index $\beta$ are consistent with being the same. The inner index $\alpha$ and the beta model core radius $r_{c}$ are statistically different, but this is expected because of the cluster's ellipticity. The brightness profile and the the best-fit model for NW1 are shown in Figure 4(b) (The NW2 profile is not shown, as the fit is good, and there is nothing special about it.) Notably, the NW1 brightness profile shows a $4 \sigma$ dip- $-25 \%$ below the model-8-10 kpc behind the front. This feature is seen in the image in Figure 4(a) (on the continuation of the right arrow). Along with the boxy shape (left arrow), it looks just like the deformations expected from KHIs (e.g., Roediger et al. 2013a, see their Figure 6) and seen in a few other clusters. In particular, multiple edges would be the $\mathrm{KH}$ eddies that develop along the LOS. 


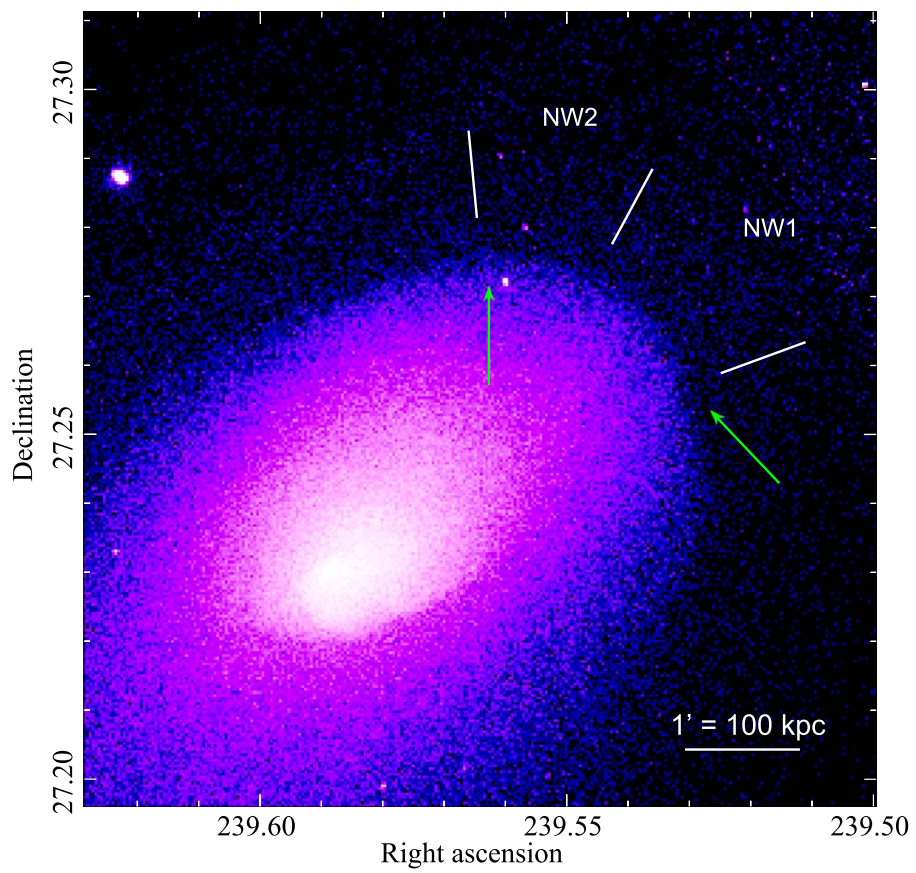

(a)

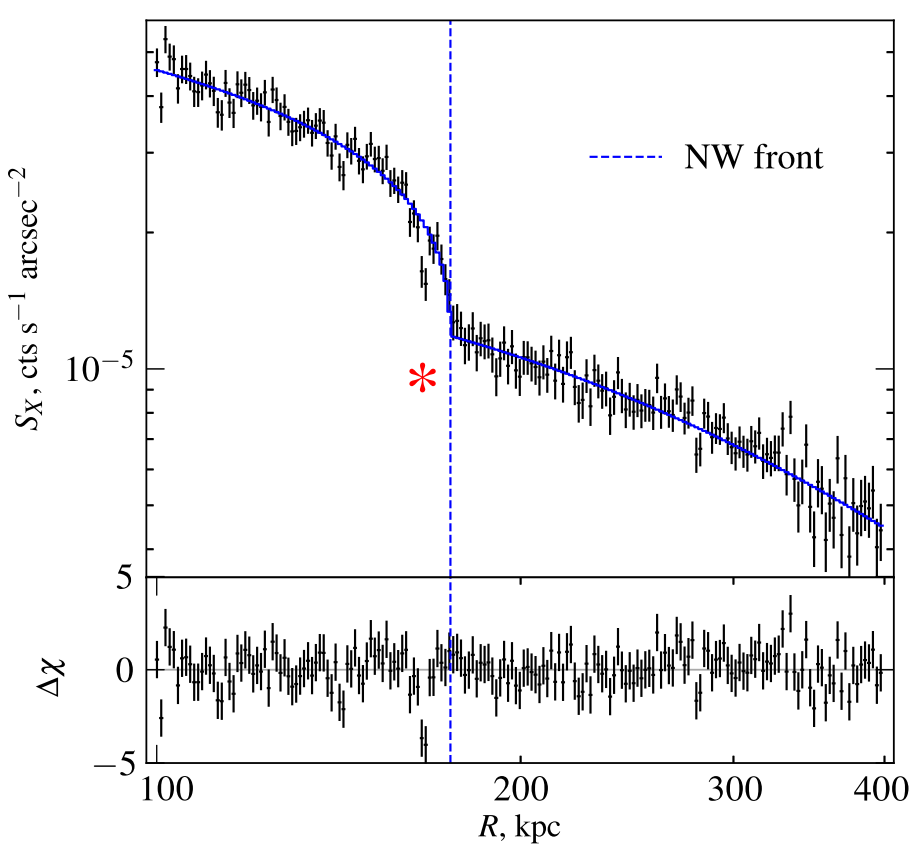

(b)

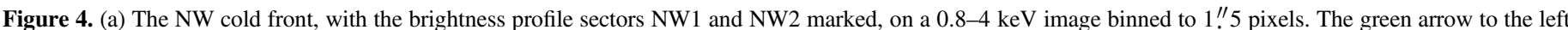

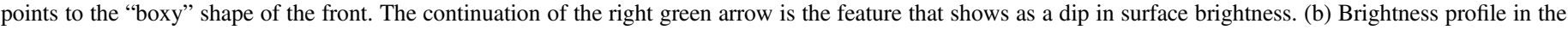

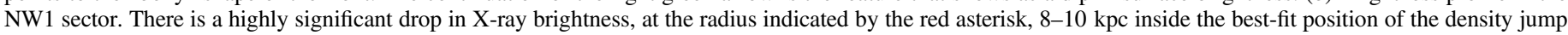
(dashed line). The best-fit positions are statistically identical in the two sectors.
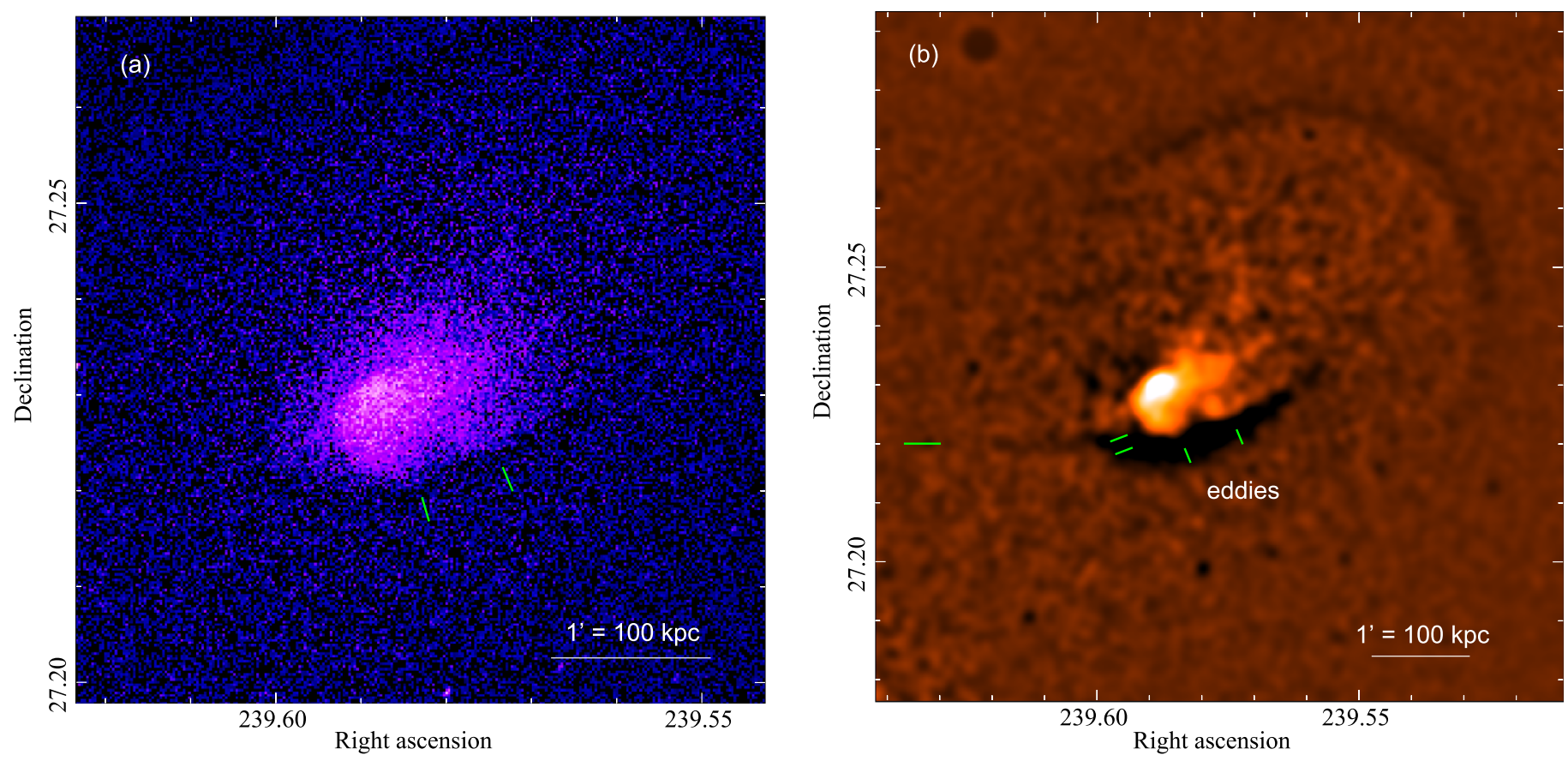

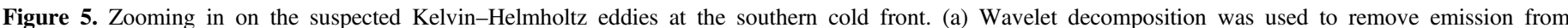

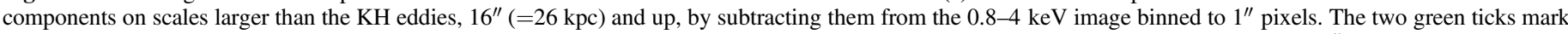

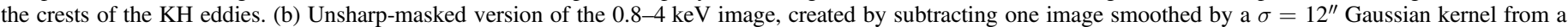

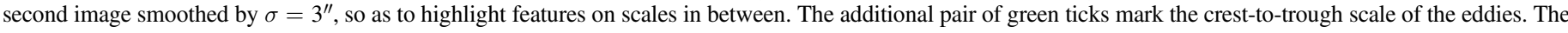
horizontal green line to the left points along the channel discussed in Section 5 .

\section{Constraints on Plasma Viscosity}

Even without any image enhancements, the X-ray image of the southern front (Figures 1 and 2(b)) shows a wavy structure that looks like the classic KHI at the interface of two gas layers with velocity shear. In Figure 5(a), we show a slightly enhanced image of the small-scale structure by subtracting the large-scale $(\geqslant 26 \mathrm{kpc})$ wavelet components from the raw image. In Figure 5(b), we instead apply the usual unsharp mask. Both images reveal two prominent bumps of the cold 
front surface that we interpret as two developed $\mathrm{KH}$ eddies, spaced by $55 \mathrm{kpc}$, with a crest-to-trough amplitude of 13-15 kpc (green dashes in Figure 5(b)). This amplitude is a lower limit because projection can only make it look smaller. The high contrast of the edge suggests that we are getting an edge-on view of the shear layer. This is only the second cold front that affords us a good, direct, and unambiguous view of the KH eddies; the other one is A3667 (Vikhlinin 2011; Ichinohe et al. 2017).

If these are indeed $\mathrm{KH}$ eddies, they present an opportunity to constrain the ICM effective viscosity. In our picture, the gas inside the southern cold front is flowing along the curved edge from the NW and spirals inward with the velocity that we estimated in Section 3.2, while the outer gas has a negligible velocity. R13 performed a numerical study of the growth of KHIs on cluster cold fronts for a range of values of isotropic viscosity (under the assumption of no magnetic fields) both Spitzer-like with strong temperature dependence as well as temperature-independent. They covered a range of gas parameters that included the A2142 southern front-in fact, they used it as one of their fiducial cases (using the early M00 results that did not show the eddies). While the R13 simulations are $2 \mathrm{D}$, they should provide a good qualitative approximation for the flow geometry expected at the cold front. Thus, all we need is to find where our new results fit in the R13 study to derive an estimate of the viscosity under their assumptions. We will try to constrain the isotropic Spitzer-like viscosity.

A full Spitzer viscosity would suppress the growth of KHI on small scales, so that only the perturbations of the interface between the two fluids larger than a critical wavelength can grow (R13, their Equation (28)):

$$
\begin{aligned}
\lambda_{\text {crit }}= & 70 \mathrm{kpc}\left(\frac{\operatorname{Re}_{\text {crit }}}{30}\right)\left(\frac{U}{500 \mathrm{~km} \mathrm{~s}^{-1}}\right)^{-1} \\
& \times\left(\frac{n_{e}}{9 \times 10^{-3} \mathrm{~cm}^{-3}}\right)^{-1}\left(\frac{k T_{\mathrm{ICM}}}{9.0 \mathrm{keV}}\right)^{5 / 2},
\end{aligned}
$$

where the density and temperature are those observed on the hotter side of the front from Table 1 (because the temperature dependence of the Spitzer viscosity makes that side dominate the effect), $U$ is the relative shear velocity of the gases on two sides of the cold front, and $\mathrm{Re}_{\text {crit }}$ is a Reynolds number defined for the KHI as

$$
\operatorname{Re} \equiv \frac{\lambda U}{\nu}
$$

where $\nu$ is the kinematic viscosity. The full Spitzer viscosity is (Spitzer 1962; Sarazin 1988)

$$
\mu=6100 \mathrm{~g} \mathrm{~cm}^{-1} \mathrm{~s}^{-1}\left(\frac{k T}{9.0 \mathrm{keV}}\right)^{5 / 2}\left(\frac{\ln \Lambda}{40}\right)^{-1},
$$

where $\nu=\mu / \rho$ and $\ln \Lambda \approx 37$ for the density and temperature we measure outside the southern cold front.

Based on simulations, R13 showed that for Spitzer-like viscosity, a conservative value is $\mathrm{Re}_{\text {crit }}=30$ to suppress KHI. We do see a developed KHI, so for our wavelength, $\operatorname{Re}>30$. To place a somewhat more accurate lower limit on the Reynolds number, and thus an upper limit on the viscosity, we compare our eddies with those in the R13 simulations at a similar growth stage. Figure 8 in R13 shows the development of KHI for different Reynolds numbers and the interface parameters very close to ours (their density contrast is 2 versus our 1.9 and their $M=0.5$ versus our rough estimate of $0.36 \pm 0.12$ ). For our front, we can use the peak-to-peak distance to measure the KHI $\lambda \simeq 55 \mathrm{kpc}$. The amplitude (half of the crest-to-trough distance) appears to be at least $0.10-0.12 \lambda$. There are not enough photons to resolve the small-scale features in the eddies, such as the expected turningover of the tip of the eddy, though observers with imagination would see a hint of this in the wavelet-subtracted image.

We can estimate the time that the eddies had to grow to their present amplitude. The inviscid KH timescale (R13, their Equations (2)-(3)) is

$$
\tau_{\text {KHinvis }}=\frac{\sqrt{\Delta}}{2 \pi} \frac{\lambda}{U},
$$

where

$$
\Delta=\frac{\left(\rho_{\text {cold }}+\rho_{\text {hot }}\right)^{2}}{\rho_{\text {cold }} \rho_{\text {hot }}}
$$

is related to the growth time of the eddies estimated from $t=L / U$, by

$$
\frac{t}{\tau_{\text {KHinvisc }}}=\frac{2 \pi L}{\sqrt{\Delta} \lambda} .
$$

If we take the distance $L$ that the perturbations have traveled along the front to be from the crests of the eddies to the eastern side of the front, $L \approx 50-100 \mathrm{kpc}$ and $t \approx 3-6 \tau_{\text {KHinvisc. }}$.

If we compare our eddy amplitude to R13 at this early growth stage (see their Figure 8 and the left panel of Figure 10), they look similar to the case with $\mathrm{Re}=100$ or above and rule out Reynolds numbers much lower than that. We note that the R13 simulations assumed uniform density on each side of the interface, whereas our density increases toward the cluster center (away from the interface) and changes noticeably on the scale of the disturbance. This is likely to decrease the depth of the troughs compared to the simulated case, so the above estimate should be conservative.

To convert this to a constraint on the viscosity, Equations (4) and (5) can be combined:

$$
\begin{aligned}
\frac{\mu_{S}}{\mu}= & 5\left(\frac{n_{H}}{9 \times 10^{-3} \mathrm{~cm}^{-3}}\right)^{-1}\left(\frac{k T_{e}}{9.0 \mathrm{keV}}\right)^{5 / 2}\left(\frac{\ln \Lambda}{40}\right)^{-1} \\
& \times\left(\frac{\operatorname{Re}}{100}\right)\left(\frac{\lambda}{55 \mathrm{kpc}}\right)^{-1}\left(\frac{U}{500 \mathrm{~km} \mathrm{~s}^{-1}}\right)^{-1} .
\end{aligned}
$$

Here, we again used the values of the gas density and temperature on the hotter side of the front, the shear velocity that we estimated in Section 3.2, and the above wavelength and Reynolds number of the KHI. The velocity of the flow is the most uncertain parameter for our constraint, but even if we use a very conservative upper limit of $1500 \mathrm{~km} \mathrm{~s}^{-1}$ (Section 3.2), the viscosity should still be lower than Spitzer.

The NW front also shows hints of KHIs, including the boxy shape of the front and the apparent double density edge seen in projection (Section 3.4). They are not seen directly in the plane of the sky as the southern front eddies, so any constraints from them would be more uncertain than those above. However, the NW edge samples a factor 4 different gas density and possibly a different velocity, so it may be interesting to perform a joint study of the two edges, perhaps using hydrodynamic 


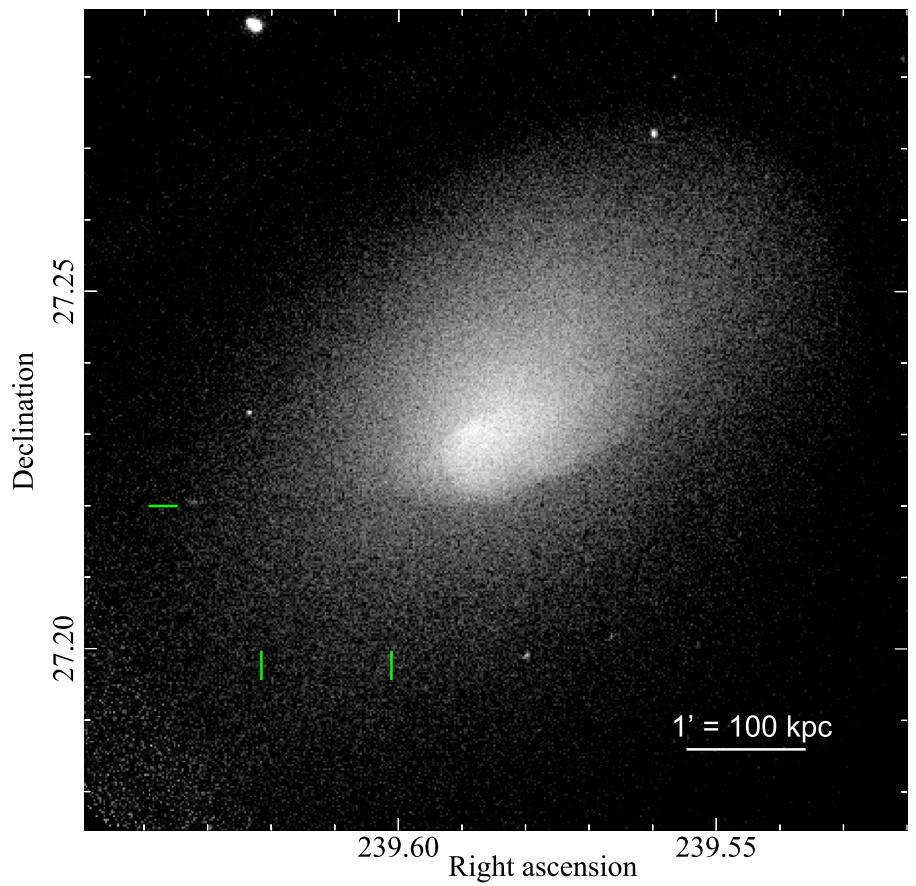

(a)

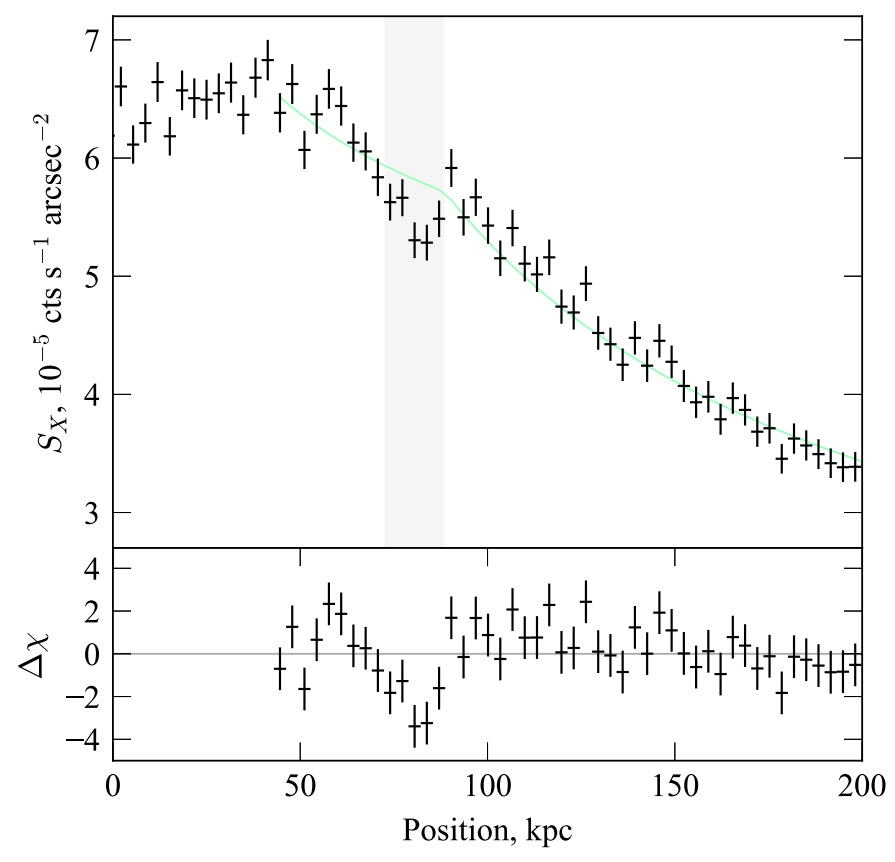

(b)

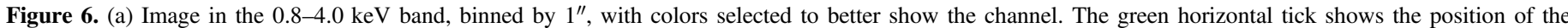

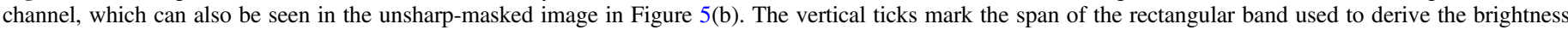

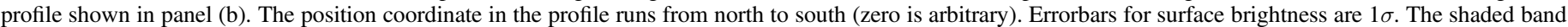

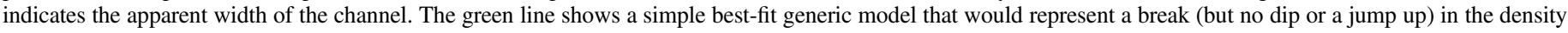
profile, and residuals in the lower panel are for this model.

simulations to reproduce their morphology and better constrain the flow velocities.

The R13 simulations have a major omission-they do not include magnetic fields, which we know are present in the intracluster plasma, and furthermore, should be significantly amplified and stretched along the cold front surface because of the expected draping (Ettori \& Fabian 2000; Vikhlinin et al. 2001a; Lyutikov 2006; Dursi \& Pfrommer 2008). ZuHone et al. (2015) showed via high-resolution MHD simulations that in the context of sloshing cold fronts in clusters, isotropic Spitzer viscosity reduced by a factor $\sim 0.1$ produces similarlooking cold fronts as the anisotropic Braginskii viscosity that describes the magnetized plasma. Thus, our estimate of the effective viscosity of $<1 / 5$ Spitzer is in agreement with full anisotropic viscosity in the presence of the magnetic fields.

\section{X-Ray Channel}

There is a subtle, long X-ray brightness channel that extends from the the middle of the southern cold front to the east. We selected the contrast in the unbinned X-ray image shown in Figure 6(a) to emphasize this linear depression. The unsharpmasked image in Figure 5(b) helps to see the feature's location. It is not a residual artifact of any ACIS chip gaps or edges, which are corrected for in all our images. Further, in this mosaic of slightly different pointings, the feature does not overlap with any chip gap or edge. Even if the exposure maps were significantly inaccurate, the amplitude of the effective exposure variations over the channel region that it corrects for is $<2 \%$, while the depth of the channel is much greater. The channel is aligned with the southern cold front (with its branch that does not curve toward the center but continues eastward,
Section 3). While the channel is most apparent to the east of the front, it may continue west, wrapping around the southern front. However, the much greater brightness gradient associated with the front there, as well as the $\mathrm{KH}$ eddies, preclude the detection of a subtle dip, because the baseline brightness is very uncertain.

While large, apparently significant deviations, some arranged in patterns, are expected in a noisy image with many independent pixels (the "look elsewhere" effect), this apparent linear feature is not found at a random place, but rather at a continuation of a prominent cold front. So it is likely to be a real structure.

We selected a section of the channel $110 \mathrm{kpc}$ long, where the channel is unobstructed by brighter features, and extracted a brightness profile across it in a strip indicated by the tick marks in Figure 6(a). The width of the channel is about $15 \mathrm{kpc}$. The brightness profile is shown in Figure 6(b), where each bin is $3.3 \mathrm{kpc}\left(2^{\prime \prime}\right)$ wide. To quantify the dip amplitude, we performed a simple fit of the brightness in the vicinity of the dip with a broken power-law model (which would represent a break in the density profile, but not allowing for a dip or a density jump up or down at the break), shown in green. There is a very significant $\sim 9 \%-12 \%$ depression in the surface brightness at the center of the channel, where two bins are each $>3 \sigma$ below the model and below the brightness in bins immediately to the right (outward).

The origin of such a density depression is not immediately clear. Simply projecting any number of monotonically declining brightness profiles of any shape would not create a brightness depression (but could create multiple brightness edges, as seen elsewhere in A2142) — as long as the density gradients point in the same general direction of the cluster 
center. One can imagine two cold fronts facing each other, with their gradients in the opposite directions, as in two subcluster cores about to collide and a low-density layer between them. However, based on the X-ray image, such a scenario is clearly not the case in A2142. Perhaps some other unexpected gas geometries could emerge in a merging cluster.

If we take the premise that the feature is indeed due to a density depression, not the presence of an edge-like profile facing the opposite direction, the geometry of this channel has to be a relatively thin sheet of lower-density gas, seen along its edge. If we consider the surface brightness profile of a NE-SW cross section of the cluster at the position of the channel, we must empty of gas the central $35 \mathrm{kpc}$ interval along the LOS to remove $10 \%$ of the flux. Since the channel cannot be completely devoid of gas, the true extent along the LOS should be significantly greater.

We have reported a similar subtle channel in the merging cluster A520 (Wang et al. 2016). There, it was aligned with an apparent direction of a secondary subcluster merger. An intriguing possibility is that these channels are examples of a plasma depletion layer (PDL) — a feature observed when the magnetic field gets stretched and amplified to values where its energy density becomes comparable to thermal pressure of its host plasma. This happens, for example, when the solar wind drapes around a planetary magnetosphere, gets amplified, and squeezes the plasma out from the narrow layer around the obstacle (Øieroset et al. 2004). A flow of magnetized plasma around a cluster cool core was simulated, e.g., by Dursi \& Pfrommer (2008), and a similar draping phenomenon was predicted. While they used a uniform magnetic field in the gas flow, a tangled field, more representative of clusters, produces a similar end result (ZuHone et al. 2013). While cold fronts are obvious locations for PDL, sheets and filaments of significantly amplified field can emerge in other locations with coherent gas flows. ZuHone et al. (2011) presented MHD simulations of a sloshing core and traced the evolution of the magnetic fields. In their Figure 23, there is a particularly illuminating example of a plasma depletion phenomenon. A filament of an amplified magnetic field aligned with the cold front, but located at a distance from it, is in pressure equilibrium with the surrounding gas, but because the pressure contribution from the amplified magnetic field is significant $(30 \%$ of thermal pressurecompared to the usual $\sim 1 \%$ ), its thermal pressure is reduced by that amount essentially by squeezing the gas from the filament. This would produce an X-ray feature just like the channel we see aligned with the cold front in A2142. Our channel is located well within the sloshing region delineated by the NW cold front, and coherent gas flows are easily expected throughout this region. A possibly similar feature, though seen as an enhancement rather than a depression in X-ray brightness, was reported near the cold front in the Virgo core (Werner et al. 2016b).

The existence of such layers of draped magnetic fields around cold fronts have long been proposed to explain the suppressed thermal conduction and diffusion across the front and the front stability (Ettori \& Fabian 2000; Vikhlinin et al. 2001a; MV07). The KHIs at the southern front (Section 4) allowed us to evaluate the effective ICM viscosity. If the layer that we see in A2142 indeed has an amplified and ordered field and wraps around the southern front, it is the likely underlying physical mechanism that regulates the growth of those KHIs and determines that effective viscosity.

\section{Summary}

A2142 provides a laboratory to study several interesting effects in the intracluster plasma and in cluster cool cores. It exhibits four cold fronts-three in the core (two of which were the initial discovery of cold fronts in M00) and one $1 \mathrm{Mpc}$ from the center, indicating long-lived sloshing set off by a strong disturbance from a merger. In this work, we have studied the three inner fronts using a $200 \mathrm{ks}$ Chandra data set. For the southern front, we estimate the velocity of the tangential gas flow inside the front from an estimate of the centripetal acceleration and obtain $v=550 \pm 190 \mathrm{~km} \mathrm{~s}^{-1}(M=0.36 \pm 0.12$ w.r.t. the sound speed in the gas on the hotter side of the front). The southern front is clearly disrupted by $\mathrm{KH}$ instability, exhibiting two eddies separated by $55 \mathrm{kpc}$ with an amplitude of $6-7 \mathrm{kpc}$. This is only the second reported example of the clearly observed $\mathrm{KH}$ eddies in the plane of the sky (the other one is A3667; other reports of the KHI were based on interpreting the structure in the front brightness profiles as eddies in projection). We compare the observed eddies with the numeric study of the growth of $\mathrm{KHI}$ in the context of cluster cold fronts by Roediger et al. (2013b), who included isotropic viscosity in their simulations. The A2142 eddies match the simulations if the isotropic, Spitzer-like viscosity is suppressed by a factor at least 5 . The velocity of the gas flow is the biggest uncertainty in this estimate, but the viscosity has to be lower than Spitzer even if we assume a $M=1$ flow. From the numeric comparison of the effects of isotropic Spitzer viscosity and anisotropic Braginskii viscosity in the presence of gas sloshing and stretching of the magnetic fields (ZuHone et al. 2015), such a suppressed effective isotropic viscosity is consistent with full Braginskii anisotropic viscosity. Our viscosity constraints are in line with several recent results for other clusters based on the KHI at cold fronts (Roediger et al. 2013a; Ichinohe et al. 2017; Su et al. 2017) as well as on the observed details of gas stripping for an infalling galaxy (Kraft et al. 2017).

A2142 has a cool, dense peak, whose specific entropy index $\left(K \approx 49 \mathrm{keV} \mathrm{cm}^{2}\right)$ makes it a rare "warm core," an intermediate case between the cool cores with sharply peaked, lowentropy cores and non-cool-core clusters with flat cores. The peak is offset from the BCG by $30 \mathrm{kpc}$. Once the cool sloshing structure (that includes this peak, the inner cold front, and the southern cold front) is approximately subtracted using wavelet decomposition, we see that the larger-scale emission in the core is well centered on the BCG, confirming the lensing result (Okabe \& Umetsu 2008) that the BCG is at the center of the cluster gravitational potential. This is the largest observed offset between the cool peak and the center of the potential for any cluster that still exhibits a well-defined peak. The extreme sloshing in A2142 should have displaced the former cool core from the center of the potential, which facilitated its disruption, as simulated in ZuHone et al. (2010). The displaced peak expands, loses the stability provided by gravity, and becomes more susceptible to sloshing-induced mixing with the hotter gas. The BCG does not show a strong AGN (exhibiting only a very weak radio source) and there is no evidence for X-ray cavities, suggesting that the displaced peak has starved its nucleus of the accreting gas for a significant period.

Finally, we detect an intriguing "channel" in the X-ray brightness, $>100 \mathrm{kpc}$ long, $\sim 15 \mathrm{kpc}$ wide, with a $\sim 10 \%$ dip in brightness, that appears to be aligned with the southern cold front. It is similar to the channel we observed in A520 (Wang et al. 2016) (though that channel is aligned with the axis of a secondary merger, not with a cold front). The channel should be a sheet of 
low-density gas seen edge-on. While some non-obvious 3D gas distributions cannot be excluded based on the X-ray image of this merging cluster, we think that a plausible explanation of this feature is a PDL. In such a layer, the stretched and amplified magnetic field in the sloshing core may reach a pressure comparable with the thermal pressure of the gas, squeezing the gas from the layer. Such phenomena are observed when the solar wind flows around an obstacle, and also seen in simulations of sloshing cluster cores that include magnetic fields (ZuHone et al. 2011). Such channels may provide an interesting additional tool to study the intracluster magnetic fields.

We thank the referee for useful criticism and detailed comments. Q.H.S.W. was supported by Chandra grants GO3-14144Z, AR5-16013X and GO8-19114.

\section{ORCID iDs}

Qian H. S. Wang (10) https://orcid.org/0000-0001-8468-9164 Maxim Markevitch (1) https://orcid.org/0000-0003-0144-4052

\section{References}

Anders, E., \& Grevesse, N. 1989, GeCoA, 53, 197

Ascasibar, Y., \& Markevitch, M. 2006, ApJ, 650, 102

Buote, D. A., Humphrey, P. J., \& Stocke, J. T. 2005, ApJ, 630, 750

Cavagnolo, K. W., Donahue, M., Voit, G. M., \& Sun, M. 2009, ApJS, 182, 12

Churazov, E., Forman, W., Jones, C., \& Böhringer, H. 2003, ApJ, 590, 225

Clarke, T. E., Blanton, E. L., \& Sarazin, C. L. 2004, ApJ, 616, 178

Dupke, R., White, R. E., III, \& Bregman, J. N. 2007, ApJ, 671, 181

Dursi, L. J., \& Pfrommer, C. 2008, ApJ, 677, 993

Ettori, S., \& Fabian, A. C. 2000, MNRAS, 317, L57

Ettori, S., Gastaldello, F., Gitti, M., et al. 2013, A\&A, 555, A93

Ghizzardi, S., Rossetti, M., \& Molendi, S. 2010, A\&A, 516, A32

Giacintucci, S., Markevitch, M., Cassano, R., et al. 2017, ApJ, 841, 71

Hamer, S. L., Edge, A. C., Swinbank, A. M., et al. 2012, MNRAS, 421, 3409

Hickox, R. C., \& Markevitch, M. 2006, ApJ, 645, 95

Ichinohe, Y., Simionescu, A., Werner, N., \& Takahashi, T. 2017, MNRAS, 467, 3662

Johnson, R. 2011, PhD thesis, Dartmouth College

Kalberla, P. M. W., Burton, W. B., Hartmann, D., et al. 2005, A\&A, 440, 775

Keshet, U., Markevitch, M., Birnboim, Y., \& Loeb, A. 2010, ApJL, 719, L74

Kraft, R. P., Roediger, E., Machacek, M., et al. 2017, ApJ, 848, 27

Lyutikov, M. 2006, MNRAS, 373, 73

Machacek, M., Dosaj, A., Forman, W., et al. 2005, ApJ, 621, 663
Markevitch, M., Bautz, M. W., Biller, B., et al. 2003, ApJ, 583, 70

Markevitch, M., Gonzalez, A. H., David, L., et al. 2002, ApJL, 567, L27

Markevitch, M., Ponman, T. J., Nulsen, P. E. J., et al. 2000, ApJ, 541, 542

Markevitch, M., \& Vikhlinin, A. 2007, PhR, 443, 1

Markevitch, M., Vikhlinin, A., \& Mazzotta, P. 2001, ApJL, 562, L153

Mazzotta, P., Fusco-Femiano, R., \& Vikhlinin, A. 2002, ApJL, 569, L31

Mazzotta, P., Markevitch, M., Vikhlinin, A., et al. 2001, ApJ, 555, 205

Million, E. T., Allen, S. W., Werner, N., \& Taylor, G. B. 2010, MNRAS, 405,1624

Oegerle, W. R., Hill, J. M., \& Fitchett, M. J. 1995, AJ, 110, 32

Øieroset, M., Mitchell, D. L., Phan, T. D., et al. 2004, SSRv, 111, 185

Okabe, N., \& Umetsu, K. 2008, PASJ, 60, 345

Owers, M. S., Nulsen, P. E. J., Couch, W. J., \& Markevitch, M. 2009, ApJ, 704, 1349

Roediger, E., Brüggen, M., Simionescu, A., et al. 2011, MNRAS, 413, 2057

Roediger, E., Kraft, R. P., Forman, W. R., Nulsen, P. E. J., \& Churazov, E. 2013a, ApJ, 764, 60

Roediger, E., Kraft, R. P., Nulsen, P., et al. 2013b, MNRAS, 436, 1721

Roediger, E., Lovisari, L., Dupke, R., et al. 2012, MNRAS, 420, 3632

Rossetti, M., Eckert, D., De Grandi, S., et al. 2013c, A\&A, 556, A44

Sarazin, C. L. 1988, X-ray Emission from Clusters of Galaxies (Cambridge: Cambridge Univ. Press)

Simionescu, A., Werner, N., Forman, W. R., et al. 2010, MNRAS, 405, 91

Simionescu, A., Werner, N., Urban, O., et al. 2012, ApJ, 757, 182

Spitzer, L. 1962, Physics of Fully Ionized Gases (2nd ed.; New York: Interscience)

Su, Y., Kraft, R. P., Roediger, E., et al. 2017, ApJ, 834, 74

Tittley, E. R., \& Henriksen, M. 2005, ApJ, 618, 227

Venturi, T., Rossetti, M., Brunetti, G., et al. 2017, A\&A, 603, A125

Vikhlinin, A. 2011, in Structure in Clusters and Groups of Galaxies in the Chandra Era ed., ed. J. Vrtilek \& P. J. Green (Chandra X-ray Center), 53

Vikhlinin, A., Forman, W., \& Jones, C. 1994, ApJ, 435, 162

Vikhlinin, A., Markevitch, M., \& Murray, S. S. 2001a, ApJ, 551, 160

Vikhlinin, A., Markevitch, M., \& Murray, S. S. 2001b, ApJL, 549, L47

Vikhlinin, A., Markevitch, M., Murray, S. S., et al. 2005, ApJ, 628, 655

Vikhlinin, A., McNamara, B. R., Forman, W., et al. 1998, ApJ, 502, 558

Walker, S. A., Hlavacek-Larrondo, J., Gendron-Marsolais, M., et al. 2017, MNRAS, 468, 2506

Walker, S. A., Sanders, J. S., \& Fabian, A. C. 2016, MNRAS, 461, 684

Wang, Q. H. S., Markevitch, M., \& Giacintucci, S. 2016, ApJ, 833, 99

Werner, N., Zhuravleva, I., Canning, R. E. A., et al. 2016a, MNRAS, 460, 2752

Werner, N., ZuHone, J. A., Zhuravleva, I., et al. 2016b, MNRAS, 455, 846

ZuHone, J. A., Kunz, M. W., Markevitch, M., Stone, J. M., \& Biffi, V. 2015, ApJ, 798, 90

ZuHone, J. A., Markevitch, M., Brunetti, G., \& Giacintucci, S. 2013, ApJ, 762, 78

ZuHone, J. A., Markevitch, M., \& Johnson, R. E. 2010, ApJ, 717, 908

ZuHone, J. A., Markevitch, M., \& Lee, D. 2011, ApJ, 743, 16 